\title{
Unusual congenital coronary anomaly and myocardial ischaemia
}

\author{
D A Pascual, F Soria, $M$ Valdes
}

\begin{abstract}
Angiography was used to diagnose a rare congenital coronary anomaly with myocardial ischaemia in a woman with typical angina. All three coronary arteries arose from a solitary coronary ostium in the right aortic sinus; the left anterior descending coronary artery followed a septal course, the circumflex coronary artery ran behind the ascending aorta, and the right coronary artery followed a normal course. No significant coronary lumen narrowing was found. Transoesophageal echocardiography confirmed the anomalous origin and course of the aberrant coronary arteries. An exercise test reproduced angina, and ECG changes and myocardial perfusion study showed an anterior reversible defect. In contrast to previous reports, myocardial ischaemia was associated with the septal (intramuscular) course of the left anterior descending coronary artery; there was no other significant coronary artery disease.

(Heart 1999;82:e7)
\end{abstract}

Keywords: congenital heart defects; myocardial ischaemia; angiography; echocardiography
In the largest series of patients reported, the overall angiographical incidence of congenital coronary anomalies was $1.3 \% .^{1}$ Although most anomalies are benign and asymptomatic, some-for example, those of pulmonary artery origin or those following an interarterial course (between the aorta and pulmonary trunk), are associated with myocardial ischaemia, ventricular arrhythmias, syncope, or sudden death; therefore early recognition is vital. A solitary coronary ostium in the aorta, in the absence of other major congenital cardiovascular anomalies (persistent truncus arteriosus, tetralogy of Fallot, pulmonary atresia) is rare; in several large series, incidence ranged from $0.024 \%$ to $0.066 \% .^{1-3}$ The abnormal origin and distribution of the vessels of such an anomaly can be of a variety of subtypes.

We report the first description of a subtype of solitary coronary ostium in the right aortic sinus, with the circumflex coronary artery following a retroaortic course and the left anterior descending (LAD) coronary artery following a septal course, diagnosed by angiography and echocardiography; this anomaly presented with documented myocardial ischaemia attributed to the septal (intramyocardial) course of the LAD coronary artery, in the absence of significant coronary disease.
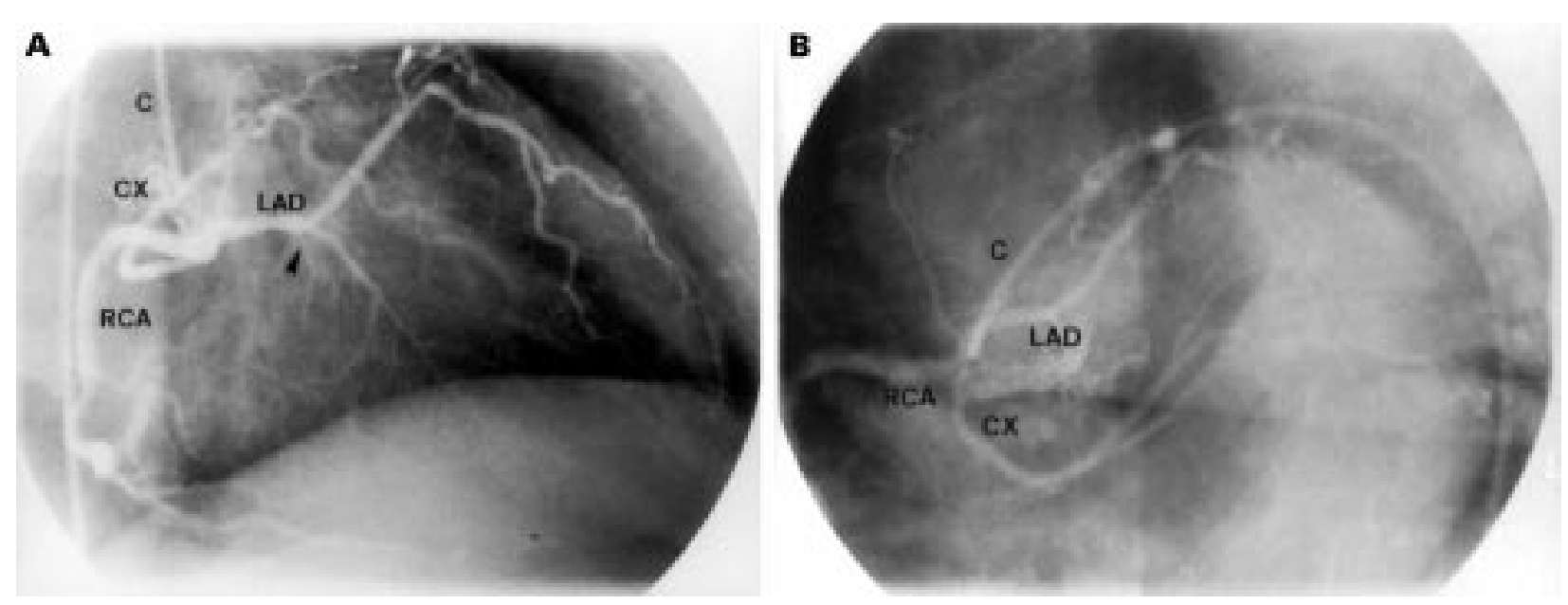

Figure 1 Coronary angiogram showing $(A)$ right anterior oblique view of the course of all three coronary arteries: the initial course of the left anterior descending coronary artery forms a caudal anterior loop and gives rise to one major septal branch, both following a septal course (arrow). The circumflex coronary artery forms a caudal posterior loop following a retroaortic course; the dominant right coronary artery follows a normal course. (B) Left anterior oblique caudal view showing all three major epicardial coronary arteries rising from a single coronary ostium. C, catheter; CX, circumflex coronary artery; $L A D$, left anterior descending coronary artery; $R C A$, right coronary artery. 


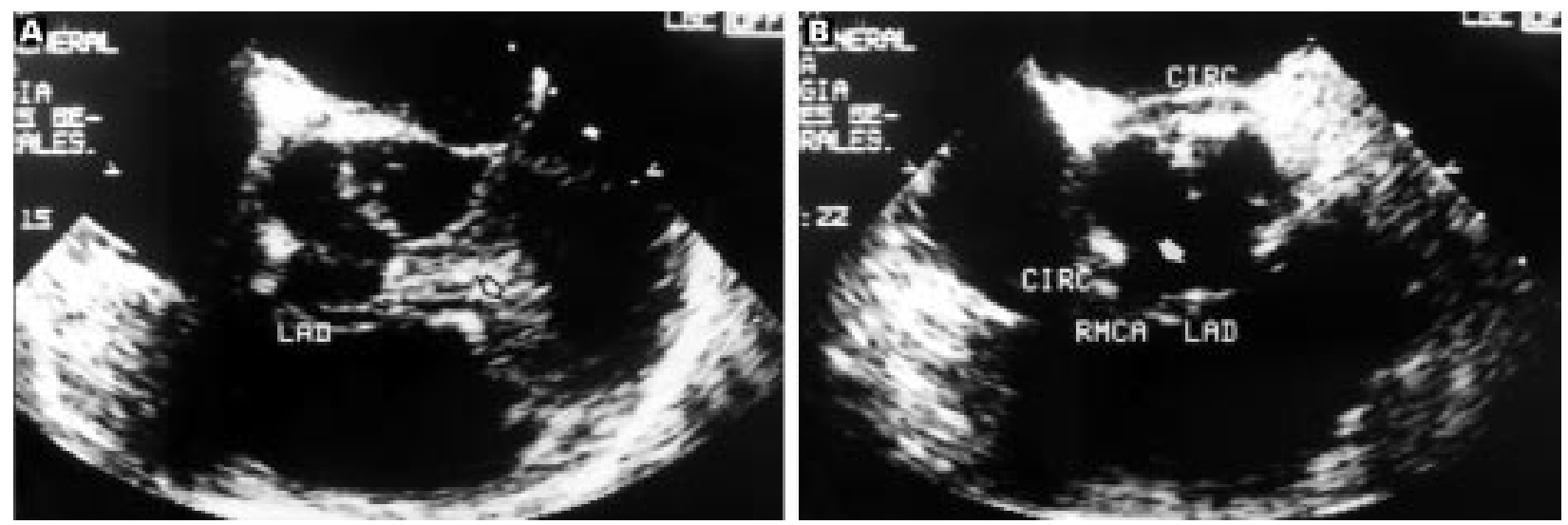

Figure 2 Transoesophageal echocardiogram showing the modified five chambers long axis view. (A) Intraseptal course (arrow) of the LAD coronary artery from the single coronary ostium. (B) Modified short axis view shows all three coronary arteries arising from a single coronary ostium in the right aortic sinus (arrow), and the retroaortic course of the circumflex. CIRC, circumflex coronary artery; LAD, left anterior descending coronary artery; $R M C A$, right main coronary artery.

\section{Case report}

A 66 year old woman presented following an episode of exercise induced typical angina, accompanied by transient ST segment depression from V3 through V6. There was no history of risk factors for coronary artery disease. Identical symptoms and ECG changes could be reproduced during a standard Bruce protocol exercise test. Transthoracic echocardiography showed isolated septal hypertrophy without subvalvar pressure gradient; no other abnormalities were noted.

Coronary angiography revealed a solitary coronary ostium at the right sinus of Valsalva, which gave rise to all three coronary arteries. The path of the right coronary artery was normal but the LAD coronary artery and circumflex coronary artery followed anomalous courses (fig 1). As in previously described angiographical criteria, ${ }^{45}$ the LAD coronary artery was bent down and forward, and passed intramuscularly through the interventricular septum, across the floor of the right ventricular outflow tract and, after giving rise to a septal branch, took an epicardial position at the midinterventricular groove (caudal and anterior loop in the right anterior oblique view). The
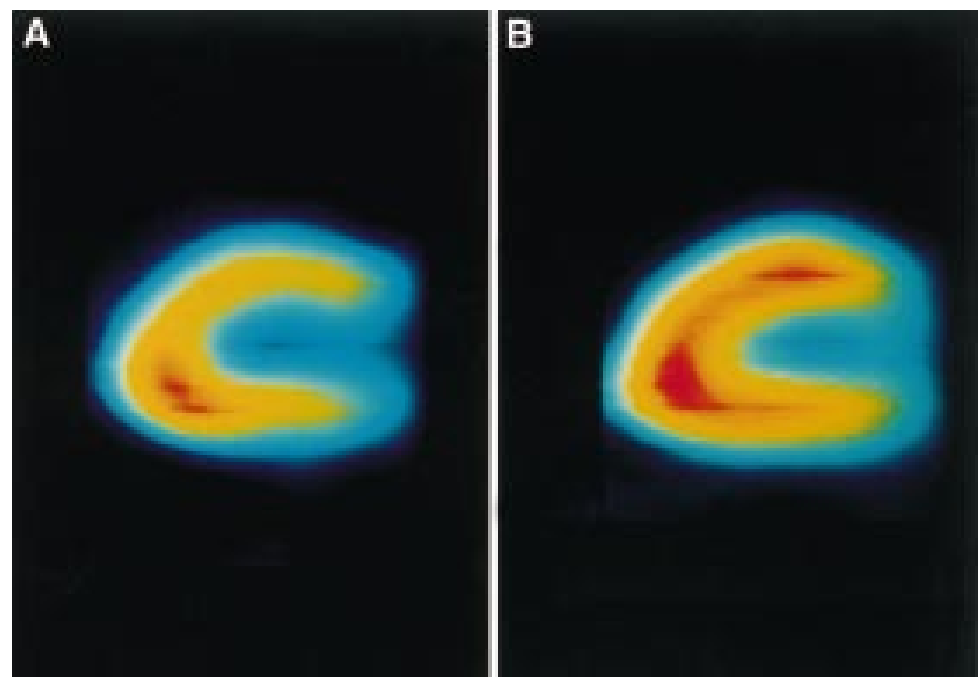

Figure 3 SPECT TT ${ }^{201}$ myocardial perfusion study showing the exercise vertical long axis slice. (A) Anterior perfusion defect which (B) shows total reversibility at rest. circumflex coronary artery followed a retroaortic course before reaching the atrioventricular groove (caudal and posterior loop in the right anterior oblique view). Despite several additional angiographical projections, no significant luminal narrowing was documented.

Transoesophageal echocardiography confirmed the presence of a solitary coronary ostium in the right sinus of Valsalva, the septal course of the LAD coronary artery, and the retroaortic course of the circumflex coronary artery (fig 2). A SPECT-T1 ${ }^{201}$ myocardial perfusion study was performed to confirm myocardial ischaemia; this also showed a reversible defect in the distribution of the LAD coronary artery (fig 3). The patient was treated with $100 \mathrm{mg}$ of atenolol daily and had no further episodes of angina pectoris.

\section{Discussion}

We report an extremely rare coronary anomaly that has been classified by Shirani and Roberts as type IID 3 of solitary coronary ostium in the aorta. ${ }^{6}$ Only two patients with the same abnormality have been described previously, when the diagnosis was made unintentionally during necropsy ${ }^{7}$; both patients had died from non-cardiovascular causes and neither patient had clinical or anatomical evidence of myocardial ischaemia or cardiac dysfunction. We have found no reports of this anomaly being diagnosed by coronary angiography during life.

In this case, myocardial perfusion study in the area of the LAD coronary artery revealed clinical ischaemia, and as we found no coronary lumen narrowing, this was attributed to the presence of the coronary anomaly. The septal (intramyocardial) course of the LAD coronary artery or the left main coronary arteries has been considered previously to be benign. ${ }^{1910} \mathrm{~A}$ review of previously published reports disclosed a total of 23 patients in whom the LAD coronary artery (eight patients) or the left main coronary artery (15 patients) arose from the right aortic sinus and thereafter followed an intramyocardial course via the ventricular septum, to the left side of the heart. ${ }^{4-13}$ In 21 of these patients, the diagnosis was made at necropsy, and in the remaining two patients by angiography. One patient had 
repeated ventricular tachycardia and died from a cardiac arrest, which could only be explained by the intramuscular course of left main coronary artery. ${ }^{12}$ Another patient, whose condition was diagnosed by angiography, had resistant ventricular tachycardia which was attributed to the intramuscular course of the left main coronary artery; the tachycardia resolved after bypass graft to the otherwise normal LAD and circumflex coronary arteries. ${ }^{13}$ The presence of cardiac events in patients in whom the left main coronary artery follows a septal course, suggests that this anomaly may be clinically significant. There was no clinical or morphological evidence of myocardial ischaemia related to the coronary anomaly in the eight patients in whom the LAD coronary artery followed an intraseptal course. However, the septal course of the LAD coronary artery may also be of importance, as our patient had ischaemia in this area. However, we could not clarify whether a true compression or a steal phenomenon were the ultimate mechanisms.

Presently, coronary angiography is used to diagnose coronary anomalies and to exclude atherosclerotic coronary disease, but transoesophageal echocardiography seems useful in the identification and confirmation of the course of the anomalous coronary arteries. ${ }^{14} 15$

In summary, we report an unusual case of solitary coronary ostium, which gave rise to the LAD and circumflex coronary arteries, which then followed anomalous courses; the septal (intramyocardial) course of the LAD coronary artery may be of clinical significance in the absence of coronary artery disease.
1 Yamanaka O, Hobbs RE. Coronary artery anomalies in 126 595 patients undergoing coronary anteriography. Cathet Cardiovasc Diagn 1990;21:28-40.

2 Desmet W, Vanhaecke J, Vrolix M, et al. Isolated single coronary artery: a review of 50000 consecutive coronary angiographies. Eur Heart f 1992;13:1637-40.

3 Lipton MJ, Barry WH, Obrez Y, et al. Isolated single coronary artery: diagnosis, angiographic classification, and clinical significance. Radiology 1979;130:39-47.

4 Ishikawa T, Brandt P. Anomalous origin of the left main coronary artery from the right anterior aortic sinus: angiographic definition of anomalous course. Am $\mathcal{f}$ Cardiol 1985;55:770-6.

5 Serota H, Barth CW, Seuc CA, et al. Rapid identification of the course of anomalous coronary arteries in adults: the dot and eye method. Am f Cardiol 1990;65:891-8.

6 Shirani J, Roberts WC. Solitary coronary ostium in the aorta in the absence of other major congenital cardiovascular anomalies. F Am Coll Cardiol 1993;21:137-43.

7 Sanes S. Anomalous origin and course of the left coronary artery in a child: so-called congenital absence of the left coronary artery. Am Heart f 1937;14:219.

8 White NK, Edwards JE. Anomalies of the coronary arteries: report of four cases. Arch Pathol 1948;45:766-71.

9 Roberts WC. Curriculum in cardiology: major anomalies of coronary arterial origin seen in adulthood. Am Heart 7 1986;111:941-61.

10 Roberts WC, Diccico BS, Waller BF, et al. Origin of the left main from the right coronary artery or from the right aortic sinus with intramyocardial tunnelling to the left side of the heart via the ventricular septum: the case against clinical significance of myocardial bridge or coronary tunnel. Am Heart F 1982;104:303-5.

11 Schulte MA, Waller BF, Hull MT, et al. Origin of the left anterior descending coronary artery from the right aortic sinus with intramyocardial tunnelling to the left side of the heart via the ventricular septum: the case against clinical significance of myocardial bridge or coronary tunnel. $\mathrm{Am}$ Heart F 1985;110:499-501.

12 Cheitlin MD, DeCastro CM, McAllister HA. Sudden death as a complication of anomalous left coronary origin from the anterior sinus of Valsalva. A not so minor congenital anomaly. Circulation 1974;50:780-7.

13 Kothari SS, Talwar KK, Venugopal P. Septal course of the left main coronary from right aortic sinus and ventricular tachycardia. Int $\mathcal{F}$ Cardiol 1998;66:207-9.

14 Fernandes F, Alam M, Smith S, et al. The role of transesophageal echocardiography in identifying anomalous coronary arteries. Circulation 1993;88:2532-40.

15 Vicente T, Lopez J, Valdes $M$. Usefulness of transoesophageal echocardiography in showing the route of anomalous coronary arteries. Heart 1996;76:183-4. 